\title{
Pediatric Immunization Terminology
}

National Cancer Institute

\section{Source}

National Cancer Institute. Pediatric Immunization Terminology. NCI Thesaurus. Code C96388.

Terminology developed by a collaborative effort of the National Cancer Institute's Enterprise Vocabulary Services, the National Institute of Child Health and Human Development, and an international group of clinicians and informaticists to support immunization in children (birth to 21 years of age). 